\title{
PERFIL SOCIODEMOGRÁFICO DE IDOSOS PARTICIPANTES DO PROGRAMA OFICINA DA MEMÓRIA NA UNIVERSIDADE ABERTA A TERCEIRA IDADE
}

Laís Alli Dias, Elaine Aparecida Lozano da Silva, Ana Caroline Rippi Moreno, Regina Celi Trindade

Camargo.

Universidade Estadual Paulista - UNESP, Departamento de Fisioterapia. Presidente Prudente. E_mail: laisalli@hotmail.com

\section{RESUMO}

Com o aumento da expectativa de vida, a queixa dos idosos frente ao seu desempenho da memoria é crescente. Assim sendo, as Oficinas de Memória realizam a prevenção e estímulo desta capacidade. O estudo teve como objetivo, caracterizar os idosos pertencentes à Oficina da Memória na Universidade Aberta da Terceira Idade (UNATI). Foi realizado estudo transversal com idosos de ambos os sexos, sendo coletados os dados sócio demográficos. A análise estatística foi realizada media e desvio padrão pelo Excel Microsoft Corporation, versão 2010. Os participantes apresentam como perfil a predominância do sexo feminino, casados, com nível de escolaridade de médio a alto, e fisicamente ativos. Estes achados permitem o conhecimento do perfil sócio demográfico de idosos que procuram auxilio de um treino para a memória, permitindo profissionais da área a se qualificarem para o atendimento desse nicho.

Palavras-chave: idoso, memoria, perfil, estimulo, prevenção.

\section{SOCIO-DEMOGRAPHIC PROFILE OF THE ELDERLY PARTICIPANTS OF THE MEMORY WORKSHOP PROGRAM AT THE OPEN SENIOR UNIVERSITY}

\begin{abstract}
With increasing life expectancy, the complaint of the elderly against the performance of memory is increasing. Thus, the Memory Workshops perform prevention and encouragement of this capacity. The study aimed to characterize the elderly belonging to the Office of Memory at the Open Senior University of the Third Age (UNATI). A cross-sectional study of elderly men and women, being collected demographic data partner. Statistical analysis was performed mean and standard deviation for Excel Microsoft Corporation, 2010. Participants version presented as profile predominantly female, married, with medium to high level of education, and physically active. These findings allow the knowledge of the socio-demographic profile of older people seeking help of a workout for the memory, allowing professionals to qualify for the service that niche.
\end{abstract}

Keywords: old, memory, profile, stimulus, prevention. 


\section{INTRODUÇÃO}

Uma das preocupações atuais que vivenciamos no Brasil é o crescente aumento de sua população idosa, que de acordo com estimativas epidemiológicas, até 2025 estará próximo a 32 milhões ${ }^{1}$.

Todo indivíduo idoso pode apresentar como fator implicante para a saúde a presença de alterações fisiológicas, bioquímicas, morfológicas e psicológicas que ocorrem de maneira gradual e progressiva no decorrer dos anos ${ }^{2}$.

Torna-se indispensável à adoção de medidas que associem a manutenção da longevidade à prevenção de doenças, reduzindo assim as internações os índices de enfermidades e mortalidade dos idosos ${ }^{3}$.

No envelhecimento também nos deparamos com alterações que levam a prejuízos da capacidade cognitiva, sendo esse um processo que ocorre em grande escala e gera maior risco ao idoso de desenvolver patologias que prejudicam a memória, tal como a Doença de Alzheimer ${ }^{4}$.

A memoria é subdividida em etapas como: a gravação, o armazenamento e a evocação de informações. O processo de envelhecimento altera esse mecanismo, resultando no declínio tanto para gravação de novas informações, quanto para lembrar as informações que fora a tempo armazenadas. Como fatores que influenciam a memoria podemos citar os fatores sócio demográficos, o estilo de vida, bem como o ambiente e o circulo de convivência do idoso ${ }^{5}$.

Os profissionais da área da saúde podem contribuir com intervenções, que atuam no desenvolvimento de técnicas de exercícios de memorização e atenção que possam auxiliar na preservação e nos declínios cognitivos ${ }^{1}$.

Nesse contexto, o objetivo do presente estudo é realizar uma caracterização dos idosos pertencentes à Oficina da Memória inserida na Universidade Aberta da Terceira Idade (UNATI).

\section{MÉTODOS}

Foi realizado estudo transversal com idosos de ambos os sexos participantes da Oficina da Memória da UNATI da FCT/UNESP de Presidente Prudente.

O estudo contou como critérios para inclusão: ter idade igual ou superior a 60 anos, ser participante da Oficina da Memória e concordar em assinar o termo de consentimento livre esclarecido. Como critério de exclusão; apresentar dificuldades de comunicação e não compreender as orientações para realização do teste estipulado.

Foi realizada coleta dos dados sócio demográficos tais como: idade, sexo, estado civil, escolaridade e nível de atividade física. A coleta foi realizada por meio de entrevista oral realizada pelo fisioterapeuta, com perguntas simples e de fácil entendimento. Para a comparação estatística foi realizado media e desvio padrão através do programa Excel (Microsoft Corporation, versão 2010).

O trabalho foi aprovado pelo Comitê de Ética e Pesquisa sob o parecer CAAE no 56496816.3.0000.5402.

\section{RESULTADOS}

O estudo foi composto por 23 idosos, com media de idade de 69,82 $\pm 5,82$ anos, com variação de 60 e 81 anos, sendo predominantemente do sexo feminino (87\%), visto na tabela 1. 
Tabela 01. Caracterização dos participantes da pesquisa segundo as variáveis idade e sexo.

\begin{tabular}{cccc}
\hline & Variáveis & Total & $\%$ \\
\hline & Idade & $69,82 \pm 5,82$ & - \\
\hline Sexo & Feminino & $20 / 23$ & $87 \%$ \\
\hline & Masculino & $03 / 23$ & $13 \%$ \\
\hline
\end{tabular}

Em relação ao estado civil foram classificados em solteiros, viúvos e casados (74\%), representando a maioria (74\%), como mostra a figura 1.

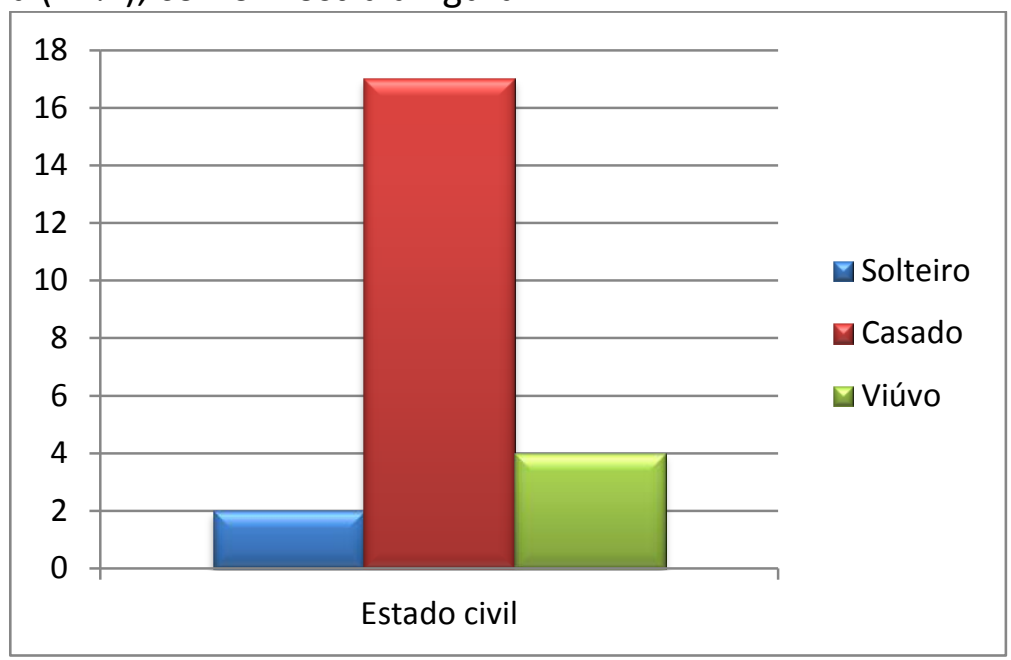

Figura 01. Caracterização dos participantes de acordo com o estado civil.

Quanto à escolaridade, foram considerados 1 grau incompleto, 1 grau completo, 2 grau incompleto, 2 grau completo e 3 grau completo, a escolaridade predominante (48\%), como observado na figura 2.

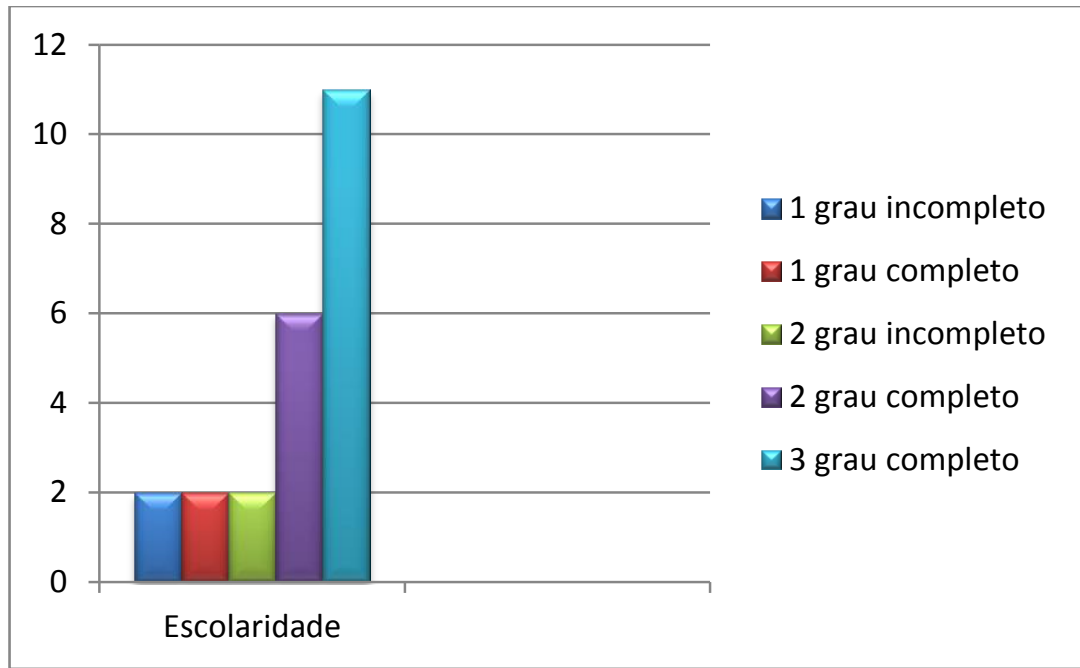

Figura 2. Caracterização dos participantes de acordo com a escolaridade.

A totalidade dos idosos que participam da Oficina da Memória foi classificada como ativa, tendo como predomínio atividades em grupo com exercícios variados, como academia, hidroginástica e programas de atividade da UNATI como Dança Sênior e Fisioterapia Preventiva.

\section{DISCUSSÃO}

As alterações cognitivas ocorrem de forma natural e irreversível, e assim como os outros tecidos do corpo humano vão perdendo a capacidade conhecida como plasticidade neuronal ${ }^{2}$. 
Alterações na cognição podem gerar quadros de demência, que segundo estatísticas crescem a cada ano, chegando a prevalência de $40 \%$ a partir dos 85 anos de idade ${ }^{6}$.

A reabilitação ou treino para a memoria é fundamental para a qualidade de vida dos idosos, proporcionam a consciência da sua nova realidade, a fim de que consigam aproveitar as habilidades ainda mantidas, visando melhorar as tarefas diárias que são de maior interesse do idoso e dos familiares ${ }^{1}$.

No presente estudo a maioria dos idosos são do sexo feminino, casados, com nível de escolaridade entre médio e alto e com nível de atividade física que os caracteriza como ativos.

Um estudo realizado com 201 idosos residentes em instituições de longa permanência, obteve também a predominância do sexo feminino ${ }^{7}$ e fato esse pode ser fundamentado pela maior expectativa de vida das mulheres no Brasil ${ }^{8}$. O crescente número de idosas no país foi considerado, como a feminização da velhice, dado esse justificado pela desigualdade de gêneros na mortalidade ${ }^{9}$.

As mulheres apresentam menor satisfação com a memoria em relação ao homem, devido a um alto senso de auto eficácia feminino, o que pode justificar a maioria de mulheres em um programa para treinamento da memória ${ }^{10}$.

Os dados de um estudo que comparou os efeitos de um treino de atenção, memoria e funções executivas na cognição de 76 idosos, corroboraram com nosso estudo pela amostra ter sido composta predominantemente de indivíduos casados e viúvos ${ }^{11}$.

O estudo sobre a Funcionalidade Familiar e Qualidade de Vida dos Idosos ${ }^{12}$, mostrou que a maioria dos participantes era casada (59,5\%), assim como o nosso que contou com $74 \%$ dos participantes. Ainda no citado estudo foi verificado que o estado civil tem relação com a qualidade de vida e que idosos casados quando comparados com solteiros e viúvos tem melhor qualidade de vida.

O uso e desuso é uma teoria bastante aceitável no caso da cognição, portanto as atividades intelectuais, como o estudo, podem atuar como um escudo para os declínios cognitivos ${ }^{11}$.Foi encontrado no presente estudo que os idosos apresentaram media de escolaridade alta, prevalecendo idosos com o 2 ㅇ e 3 ㅇ grau completo.

Um estudo dividiu idosos em dois grupos, um de idosos com baixa escolaridade e outro com maior nível de escolaridade e os submeteram a um treino cognitivo. Os dois grupos tiveram resultados positivos, porem o grupo com maior nível de escolaridade obteve melhores resultados, alinhando com outros estudos que demonstraram que idosos com maior nível de escolaridade tem um processo de cognição mais eficiente do que os com menor nível ${ }^{13}$.

No presente estudo foi constatado que $100 \%$ dos participantes são ativos, o que confirma um estudo que mostra que o número de praticantes de atividade física na terceira idade vem aumentando $^{14}$. O exercício físico proporciona a liberação de hormônios de estresse como a adrenalina, noradrenalina, ACTH, vasopressina, $\beta$-endorfina, tal evento ocasiona interferência positiva na memoria ${ }^{15}$.

Ainda relatando a eficácia do exercício físico para a memoria, um estudo comparativo com idosos sedentários e ativos demonstrou que pessoas que realizam atividades físicas frequentemente são mais resistentes ao declínio cognitivo natural quando comparados com idosos sedentários ${ }^{2}$.

\section{CONCLUSÃO}

Os participantes apresentam como perfil a predominância do sexo feminino, casados, com nível de escolaridade de médio a alto e fisicamente ativos. Estes achados permitem o conhecimento do perfil sociodemografico de idosos que procuram auxilio de um treino para a memória, permitindo profissionais da área a se qualificarem para o atendimento desse nicho. 


\section{AGRADECIMENTOS E CONFLITO DE INTERESSE}

Agradecimentos a Universidade Aberta a terceira Idade. Os autores declaram não haver qualquer potencial conflito de interesse que possa interferir na imparcialidade deste trabalho científico.

\section{REFERENCIAS}

Chariglione IPF, Janczura GA. Contribuições de um treino cognitivo para a memória de idosos institucionalizados. Psico USF.2013;18(1): 13-22. DOI:https://dx.doi.org/10.1590/S1413$\underline{82712013000100003}$

Cordeiro J, Del Castillo BL, Freitas CSD, Gonçalves MP. Efeitos da atividade física na memória declarativa, capacidade funcional e qualidade de vida em idosos. Rev. bras. geriatr. Gerontol. 2014; 17(3): 541-552. DOI : http://www.redalyc.org/articulo.oa?id=403838839008.

Vieira AAU, Aprile MR, Paulino CA. Exercício físico, envelhecimento e quedas em idosos: revisão narrativa. Revista Equilíbrio Corporal e Saúde. 2015;6(1).

Trindade APNT, Barboza MA, Oliveira FBD, Borges APO. Repercussão do declínio cognitivo na capacidade funcional em idosos institucionalizados e não institucionalizados. Fisioter Mov,2013;26(2): 281-9. DOI: http://dx.doi.org/10.1590/S0103-51502013000200005

Sato AT, Batista MPP, De Almeida MHM."Programas de estimulação da memória e funções cognitivas relacionadas": opiniões e comportamentos dos idosos participantes. Revista de Terapia Ocupacional da Universidade de São Paulo.2014; 25(1): 51-

59.DOI:http://dx.doi.org/10.11606/issn.2238-6149.v25i1p51-9

dos Reis LA, Torres GV, Novaes LKN, dos Reis LA. Deficit cognitivo como fator de risco para a limitação de atividades cotidianas em idosos institucionalizados. Revista de Psicologia.2015; 2(1).

Ferreira DC, Yoshitome AY. Prevalence and features of falls of institutionalized elders. Rev Bras Enferm. 2010;63(6):991-7. DOI: http://dx.doi.org/10.1590/S0034-71672010000600019.

Ferreira LL, Cochito TC, Caíres F, Marcondes LP, Saad PCB. 291 J Health Sci Inst. 2014;32(3):290-3.

Salgado, C. D. S. Mulher idosa: a feminização da velhice. Estudos interdisciplinares sobre o envelhecimento.2002; 4.

Pinto, JM, Neri AL. Doenças crônicas, capacidade funcional, envolvimento social e satisfação em idosos comunitários: Estudo Fibra. Cien Saude Colet.2013; 18(12): 3449-3460.DOI: http://dx.doi.org/10.1590/S1413-81232013001200002

Irigaray TQ, Gomes Filho I, Schneider RH. Efeitos de um treino de atenção, memória e funções executivas na cognição de idosos saudáveis. Psicologia: Reflexão e Crítica.2012; 25(1): 188202.DOI: http://dx.doi.org/10.1590/S0102-79722012000100023.

Andrade AINPA, Martins R. Funcionalidade familiar e qualidade de vida dos idosos. Millenium.2016; 40; 185-199. 
Fabricio AT, Silva TBLD, Kissaki PT, Vieira MGA, Ordonez TN, Wachholz TBDO, Yassuda MS. Treino cognitivo em adultos maduros e idosos: impacto de estratégias segundo faixas de escolaridade. Psico-USF.2012;17(1): 85-95.DOI: http://dx.doi.org/10.1590/S1413$\underline{82712012000100010 .}$

Siqueira GS, De Araújo CB, De Souza Barreto M. ATIVIDADE FÍSICA E TERCEIRA IDADE. Anais dos Encontros Regionais dos Estudantes de Educação Física (R3).2016; 1(1).

Fechine B, Vasconcelos O, Botelho M, Trompieri N, Carvalho J. MEMÓRIA, EXERCíCIO FÍsICO E ENVELHECIMENTO: UM ESTUDO SOBRE A RELAÇÃO EXISTENTE ENTRE A MEMÓRIA VISUOMOTORA E IDOSOS PRATICANTES E NÃO PRATICANTES DE ATIVIDADE FÍSICA. InterSciencePlace.2015;1(26).DOI: http://dx.doi.org/10.6020/1679-9844/2609 